\title{
A consulta em 7 passos Execução e análise crítica de consultas em medicina geral e familiar
}

Vítor Ramos*

\section{RESUMO}

Este artigo resume um modelo de análise crítica de consultas de medicina geral e familiar (MGF). É uma proposta de trabalho adaptável a cada situação concreta. Começou por ser, em 2005, um pequeno exercício de apoio à formação de internos da especialidade, no Centro de Saúde de Cascais. Evoluiu nos anos seguintes até à forma de livro, o qual foi editado em Setembro de 2008 integrando numerosos contributos de orientadores e de internos de MGF.

Neste modelo, cada episódio de consulta é decomposto em três fases e sete passos:

Uma fase inicial - preparação e primeiros minutos, na qual se individualizam dois passos:

1. Preparação - onde se revê a situação do médico, do consultório e do próximo paciente, antes de este ser chamado para a consulta;

2. Os primeiros minutos - onde se procede à chamada, ao encontro, ao cumprimento e ao acolhimento da pessoa, bem com à detecção de indícios físicos e emocionais, de motivos de consulta e ao acerto de agendas entre o médico e o paciente.

Segue-se uma fase intermédia com três passos: exploração, avaliação e plano (EAP), durante a qual se procede à recolha sistematizada de dados e de informação, subjectivos e objectivos, e se processam intelectualmente os dados e a informação recolhidos para chegar, sempre com a participação e o envolvimento do paciente, a uma avaliação e a um plano de acção. Consideram-se os passos:

3. Exploração - onde se recolhem, analisam e contextualizam dados e informação (subjectivos, objectivos e contextuais);

4. Avaliação - onde se fazem a interpretação, os diagnósticos, as explicações, as previsões (prognósticos) e se avaliam os impactos na qualidade de vida;

5. Plano - onde se prepara o plano de cuidados (propostas, negociação, acordos e compromissos de acção, incluindo prevenção). $\mathrm{Na}$ fase final procede-se ao encerramento da consulta e a uma reflexão:

6. Encerramento - onde se verifica se subsistem dúvidas, se revê o plano acordado e se procede ao cumprimento de despedida; 7. Reflexão e notas finais - onde se faz um breve reflexão crítica sobre o que se passou.

A organização em três fases e sete passos pode parecer fragmentária. Porém, tem objectivos de treino e de aprendizagem e tem sido aplicada de múltiplos modos para formar e aperfeiçoar atitudes e comportamentos clínicos na consulta.

Palavras-chave: Consulta; Medicina Geral e Familiar; Análise Crítica de Consultas; Formação.

\section{A CONSULTA COMO PROCESSO E COMO SISTEMA}

$\Delta$ consultaé a actividade principal do médico de família e é, também, a mais complexa. Existe vasta bibliografia sobre os ingredientes indispensáveis para realizar boas consultas, designadamente no que se refere às capacidades e às competências biomédicas, comunicacionais, relacionais e de raciocínio clínico a desenvolver. No entanto, a bibliografia especificamente dedicada à consulta, enquanto

*Médico de Família, Unidade de Saúde Familiar Marginal (Centro de Saúde de Cascais); Professor convidado da Escola Nacional de Saúde Pública/Universidade Nova de Lisboa processo estruturado, é menos numerosa. Dos autores que têm publicado sobre este processo, destacam-se Pendleton, Byrne, Livesey, Neighbour, entre outros. ${ }^{1-18}$

O presente artigo resume o livro «A Consulta em 7 Passos», editado em Setembro de 2008, que integra numerosos contributos de médicos envolvidos na formação sobre a consulta em medicina geral e familiar no internato médico desta especialidade. ${ }^{19}$

A consulta pode ser abordada como um acto discreto com antecedentes, princípio, meio, fim e consequências, que se repete milhares de vezes em cada ano de trabalho do médico de família. Por sua vez, as suas conse- 


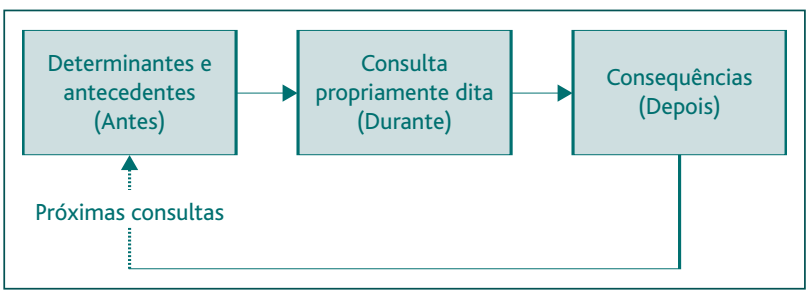

Figura 1. A consulta: processo com antecedentes, princípio, meio, fim e consequências.

quências podem ser imediatas, a médio prazo e a longo prazo, e podem passar a constituir antecedentes e determinantes de próximas consultas (Figura 1)., ${ }^{1,2}$

A consulta pode, também, ser considerada como um sistema complexo aberto, com «entradas», interacções, transformações internas, «saídas» e efeitos ou impactos. Sob esta perspectiva sistémica podem considerar-se aspectos e indicadores de contexto, de estrutura, de processo e de resultados (Figura 2).

Estamos, assim, perante um encontro de sistemas: o sistema médico e o sistema utente, ambos incluídos num sistema contextual, recebendo e emitindo influências entre si.

Em relação aos aspectos e aos indicadores de estrutura, estes dizem respeito, por exemplo, a elementos arquitectónicos e de mobiliário, disposição de cadeiras e de mesas, interposição de computador, modo de chamar os pacientes, tempos previstos, regras de marcação de consultas, etc. Incluem-se também nos aspectos de estrutura os suportes físicos dos registos clínicos (dossier clínico A4 de papel, processo clínico electrónico, etc.), bem como os múltiplos impressos e formulários em uso.

Os aspectos e indicadores de processo incidem sobre a comunicação e a relação médico-paciente, modos de condução da consulta (estruturada, por passos versus desorganizada; linear e não-linear; graus de directividade, etc.), métodos de raciocínio clínico, processos de envolvimento e participação do paciente, modos de anotar nos registos clínicos, etc.

Os indicadores de resultados podem associar-se ao conceito de critérios de sucesso da consulta, sob duas perspectivas: os objectivos do médico e os objectivos do paciente. E, todos eles, podem ser vistos a curto prazo (imediatos), a médio prazo (ou intermédios) e a longo prazo. Porém, numa consulta, apenas podemos ter con-

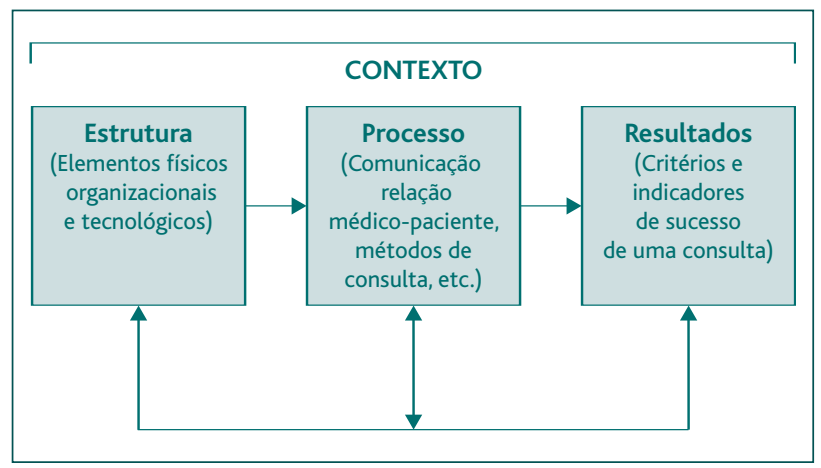

Figura 2. A consulta - sistema complexo, aberto, onde podem considerar-se: contexto, estrutura, processo e resultados.

trolo directo e, até certo ponto, garantir a estrutura e o processo. Por isso, estes devem ser exemplares. ${ }^{1-3}$

\section{MODELO CLÍNICO INTEGRADO}

A «consulta em 7 passos» tem por base um modelo clínico integrado, que é um desenvolvimento do método clínico centrado na pessoa. Isto é, centra-se na singularidade de cada pessoa e, ao mesmo tempo, presta atenção aos aspectos biomédicos, ao médico, à relação médico-paciente e ao contexto em que tudo decorre. Este modelo procura superar o modelo centrado no médico, que tende a ver o doente como objecto passivo de estudo e de intervenção. Requer um saber lidar com a complexidade da condição humana, com o vasto leque de factores que condicionam a dinâmica de saúde-doença de cada pessoa, de cada família e de grupos com necessidades especiais, numa perspectiva ecossistémica: família, contextos ocupacional, sócio-cultural e comunitário. George Engel foi pioneiro a definir e a propor esta abordagem na literatura científica internacional, no seu histórico artigo na Science, em 1977.20

As referências centrais do método clínico centrado na pessoa são as publicações da equipa de Ian McWhinney, designadamente a obra Patient-Centered Medici$n e$, de Moira Stewart e outros autores. Este método facilita a compreensão do doente e direcciona a consulta para o seu empowerment, fortalecendo a capacidade de decisão conjunta perante os problemas de cada indivíduo abordados no decorrer da consulta. ${ }^{21-23}$

Por sua vez, o modelo clínico integrado combina várias abordagens: biomédica, psicossocial, centrada na 
pessoa, atenta ao médico e à relação médico-doente, e procura equilibrá-las e harmonizá-las, sem menospre-

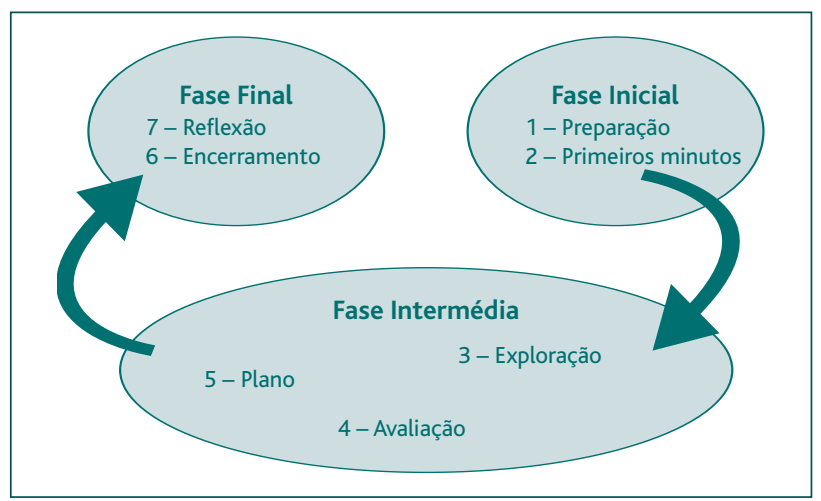

Figura 3. As três fases e os sete passos da consulta. zar nenhuma delas. Este modelo decorre da aplicação da teoria da complexidade à prática clínica, em especial à $M G F^{24-30}$

\section{FASES E PASSOS DA CONSULTA}

Propõem-se três fases e sete passos para análise de cada consulta (Figura 3 e Quadro I):

\section{- Fase inicial - preparação e primeiros minutos}

Esta fase requer um investimento na preparação do que se vai seguir. É uma fase de concentração e de focalização de atenção e de energia, individualizando-se dois passos:

Passo 1 - Preparação - onde se revê a situação do médico, do consultório e do próximo paciente, antes de este ser chamado para a consulta;

\begin{tabular}{|c|c|}
\hline Passos & Objectivos \\
\hline 1. Preparação & $\begin{array}{l}\text { a) Breve auto-avaliação do médico - forças e fraquezas para a consulta que se segue; alguma } \\
\text { necessidade fisiológica a satisfazer? Existem emoções que possam ser transportadas para a próxima } \\
\text { consulta e a vão contaminar? } \\
\text { b) Rever quem é o próximo utente - consulta breve do processo: resumo, lista de problemas, } \\
\text { terapêutica habitual, registo SOAP da última consulta, registos das últimas consultas e outra } \\
\text { informação pertinente } \\
\text { c) Avaliar sumariamente as condições do ambiente do gabinete: arejamento, limpeza, arrumação, etc. }\end{array}$ \\
\hline 2. Primeiros minutos & $\begin{array}{l}\text { Acolhimento, primeiro contacto, cumprimento. } \\
\text { Observação e escuta atentas do paciente e das suas motivações (aqui e agora) } \\
\text { Nota: a qualidade da abertura da consulta é vital para o seu sucesso. As primeiras impressões são } \\
\text { muito importantes. É nesta fase que começa a preparação do clima terapêutico. }\end{array}$ \\
\hline $\begin{array}{l}\text { 3. Exploração, análise } \\
\text { e contextualização }\end{array}$ & $\begin{array}{l}\text { Anamnese, exame objectivo e contextualização dos problemas abordados. } \\
\text { Nota: é importante procurar apreender, sem modificar, o «objecto» (a história do doente). } \\
\text { O exame físico e, em especial, a anamnese, podem ter efeitos terapêuticos (além da sua natural } \\
\text { finalidade diagnóstica). }\end{array}$ \\
\hline 4. Avaliação & $\begin{array}{l}\text { Inter-relacionar e interpretar os dados e a informação recolhidos e integrá-los num quadro explicativo } \\
\text { coerente e com significado para ambos (médico e paciente). } \\
\text { Nota: inclui a definição de problemas, a formulação de diagnósticos, aspectos de previsão } \\
\text { (prognóstico) e avaliação de impactos na funcionalidade e na qualidade de vida. }\end{array}$ \\
\hline 5. Plano & $\begin{array}{l}\text { Propor, negociar e procurar chegar a acordo sobre acções e objectivos, incluindo prevenção } \\
\text { («oportunista» ou não) }\end{array}$ \\
\hline 6. Encerramento & $\begin{array}{l}\text { Confirmar a inexistência de dúvidas, a satisfação das expectativas do paciente, acolher eventuais } \\
\text { apreciações do utente e encerrar formalmente a consulta (cumprimento de despedida). }\end{array}$ \\
\hline 7. Reflexão final & $\begin{array}{l}\text { Fase dedicada à auto-reflexão pessoal sobre a consulta e, também, para fazer a limpeza mental, } \\
\text { cognitiva e uma eventual descontaminação emocional, para passar à próxima consulta. } \\
\text { Esta fase pode ser útil para completar ou corrigir os registos clínicos referentes a essa consulta } \\
\text { e a essa pessoa. } \\
\text { Ter presente que é sempre possível melhorar! }\end{array}$ \\
\hline
\end{tabular}


Passo 2-Os primeiros minutos - onde se procede à chamada, ao encontro, ao cumprimento e ao acolhimento da pessoa, bem com à detecção de indícios físicos e emocionais, de motivos de consulta e ao acerto de agendas entre o médico e o paciente.

- Fase intermédia-exploração, avaliaçãoe plano(EAP)

Esta fase corresponde ao desenrolar da consulta, onde se faz a recolha sistematizada de dados e de informação, subjectivos e objectivos. É também nesta fase que se processam intelectualmente os dados e a informação recolhidos para chegar, sempre com a participação e envolvimento do paciente, a uma avaliação e a um plano de acção. Embora os passos devam ser sequenciais, na prática sempre ocorre alguma multidireccionalidade entre estes 3 passos. Pode acontecer, por exemplo que certos dados só se apurem ao discutir o plano, o que se traduz, depois, num andar «de diante para trás» e vice-versa. É, assim, necessário manter a disciplina na condução do processo da consulta, mas permitir a indispensável abertura e flexibilidade. Esta fase compreende três passos:

Passo 3 - Exploração - onde se recolhem, analisam e contextualizam dados e informação (subjectivos, objectivos e contextuais);

Passo 4-Avaliação-onde se fazem a interpretação, os diagnósticos, as explicações, as previsões (prognósticos) e se avaliam os impactos na qualidade de vida;

Passo 5 - Plano - onde se prepara o plano de cuidados (propostas, negociação, acordos e compromissos de acção, incluindo prevenção).

\section{- Fase final - encerramento e reflexão final}

É a altura para resumir o que se passou, para avaliar como decorreu a consulta, para verificar que se chegou ao local desejado, que não ficou nada para trás, e pensar em próximas etapas.

Consideram-se dois passos:

Passo 6 - Encerramento - onde se verifica se subsistem dúvidas, se revê o plano acordado e se procede ao cumprimento de despedida;

Passo 7 - Reflexão e notas finais - onde se faz uma breve reflexão crítica sobre o que se passou.

Esta organização em 3 fases $\mathrm{e} 7$ passos pode parecer fragmentária. Porém, tem objectivos de treino e de aprendizagem e pode ser aplicada de múltiplos modos. Pode servir, por exemplo, para aperfeiçoar atitudes e comportamentos clínicos apenas num ou em alguns dos passos e fases propostos.

Muitos dos aspectos que aqui se escalpelizam parecem coisas óbvias, feitas na prática em fracções de segundo, de modo automático, ágil e intuitivo por um médico experiente. Mas os automatismos, a agilidade e a intuição requerem um longo processo de aprendizagem e de treino. A finalidade deste exercício é ajudar e acelerar a obtenção de níveis elevados de qualidade do desempenho clínico. Infelizmente, muitos dos aspectos aqui abordados são frequentemente ignorados ou deficientemente executados, com prejuízo tanto para os pacientes, como para o próprio médico ou para a relação médico-doente.

\section{EXERCÍCIO PRÁTICO}

O exercício proposto disseca os principais processos mentais, relacionais, comunicacionais e clínicos que acontecem nas várias fases e passos de cada consulta, em especial nas consultas de complexidade média e elevada, as quais podem representar mais de metade das consultas de MGF. O exercício pode ser aplicado de várias formas:

a) pelo próprio médico, como exercício de reflexão e de auto-avaliação, escolhendo uma consulta que preveja mais complexa, podendo abranger a totalidade dos passos, ou apenas um ou mais passos que queira analisar melhor (recomenda-se a aplicação no final da consulta, com reserva de algum tempo para esse efeito);

b) por um observador externo, abrangendo apenas um ou mais passos de uma consulta ou de um número escolhido de consultas;

c) para preparação e análise de uma consulta video-gravada - autoscopia;

d) para avaliação abreviada de uma consulta na sua totalidade (ver grelha de avaliação qualitativa sumária - Anexo I);

e) para treino e reflexão dos internos de MGF durante a execução das suas consultas, aplicando-o de modo flexível, consoante as necessidades da sua formação; f) de todas as formas imaginativas que se quiser...

Passo 1 - Preparação da consulta "O começo é a parte mais importante de qualquer trabalho»

Platão 


\section{QUADRO II. Passo 1 - Preparação da consulta}

\begin{tabular}{l|l} 
Exercício & $\begin{array}{l}\text { - Auto-avaliar as condições objectivas e subjectivas do médico. } \\
\text { - Rever quem é o próximo paciente e quais os aspectos essenciais a ter presentes: ver o resumo, a lista de } \\
\text { problemas e as anotações da última consulta, em especial o Plano acordado e as notas prospectivas. } \\
\text { - Verificar sumariamente as condições físicas, de arrumação, arejamento e conforto do gabinete. }\end{array}$ \\
$\begin{array}{l}\text { Aspectos a ter } \\
\text { em conta }\end{array}$ & $\begin{array}{l}\text { Quanto ao médico } \\
\text { - Estou preparado física e emocionalmente para passar à próxima consulta? } \\
\text { Quanto ao paciente } \\
\text { - Identificação: } \\
\text { - nome e eventuais títulos pelos quais o paciente gosta de ser tratado. } \\
\text { - Contexto familiar e particularidades a ter em conta (doenças em familiares, etc.). } \\
\text { - Resumo da situação clínica e lista de problemas. } \\
\text { - Qlguns «alertas» a ter em conta. } \\
\text { - Quais os objectivos acordados? } \\
\text { - Aspecto(s) que tenha(m) ficado pendente(s) do ou dos últimos contactos. } \\
\text { Quanto ao contexto e ambiente do gabinete } \\
\text { - Ambiente (temperatura, luminosidade, etc.) e arrumação. } \\
\text { - Necessidade de arejar o consultório? De proceder a alguma limpeza? } \\
\text { - Necessidade de preparar algum material específico para a consulta com o próximo doente? }\end{array}$ \\
\hline
\end{tabular}

Começar bem uma consulta significa prepará-la cuidadosamente. A preparação da consulta visa relembrar e responder a um conjunto de perguntas, em especial, no que respeita ao doente, caso seja já uma consulta seguinte para ele. Esta preparação de cada consulta incide em três pólos (Quadro II):

- médico e o seu estado físico e emocional,

- o paciente,

- o contexto e o ambiente do gabinete, entre outros aspectos.

\section{Passo 2 - Os primeiros minutos}

A qualidade da abertura da consulta é vital para o seu sucesso. As primeiras impressões são muito importantes. É neste passo que começa a preparar-se um clima terapêutico e onde se clarificam e acordam os problemas a abordar na consulta (Quadro III). ${ }^{31,32}$

Os principais objectivos deste passo são:

a) Abrir a consulta e o processo de comunicação;

b) Captar eventuais indícios de algo latente;

c) Preparar a criação de uma relação terapêutica;

d) Esclarecero(s) motivo(s) de consulta mais importantes;

e) Acordar os problemas a abordar na consulta - esta- belecer a ordem de trabalhos da consulta, para que ambos saibam o que há para abordar, podendo verificar o que foi feito e o que falta fazer. ${ }^{32,33}$

A pergunta-chave deste passo é: - por que é que este doente veio consultar-me hoje?

É indispensável compreender o que trouxe uma pessoa a uma dada consulta, naquela altura: o(s) motivo(s) expresso(s) espontaneamente ou, momentos mais tarde, os motivo(s) adicionais expressos após solicitação não directiva da(o) médica(o). Perguntas como, por exemplo: «existe mais alguma razão para ter vindo à consulta?»; ou «há mais alguma coisa que o preocupe?» ou «precisa de mais alguma coisa?» podem ajudar a esclarecer motivos latentes, ou razões que estejam por detrás da vinda à consulta, designadamente «motivos de motivos» de consulta.

É também altura de identificar quais as ideias e as expectativas do paciente. Esta clarificação inicial permite, depois, uma melhor negociação do plano e o acordo e compromisso quanto aos objectivos e metas a atingir e à sua definição em termos realistas. A pergunta: «-O que espera de mim?» serve tanto ao médico como ao doente. 


\section{QUADRO III. Passo 2 - Os primeiros minutos da consulta}

\begin{tabular}{|c|c|}
\hline Exercício & $\begin{array}{l}\text { - Observar atentamente, explorar e analisar criticamente os aspectos e factos associados à fase inicial } \\
\text { da consulta e os motivos de consulta. }\end{array}$ \\
\hline $\begin{array}{l}\text { Aspectos a ter } \\
\text { em conta }\end{array}$ & 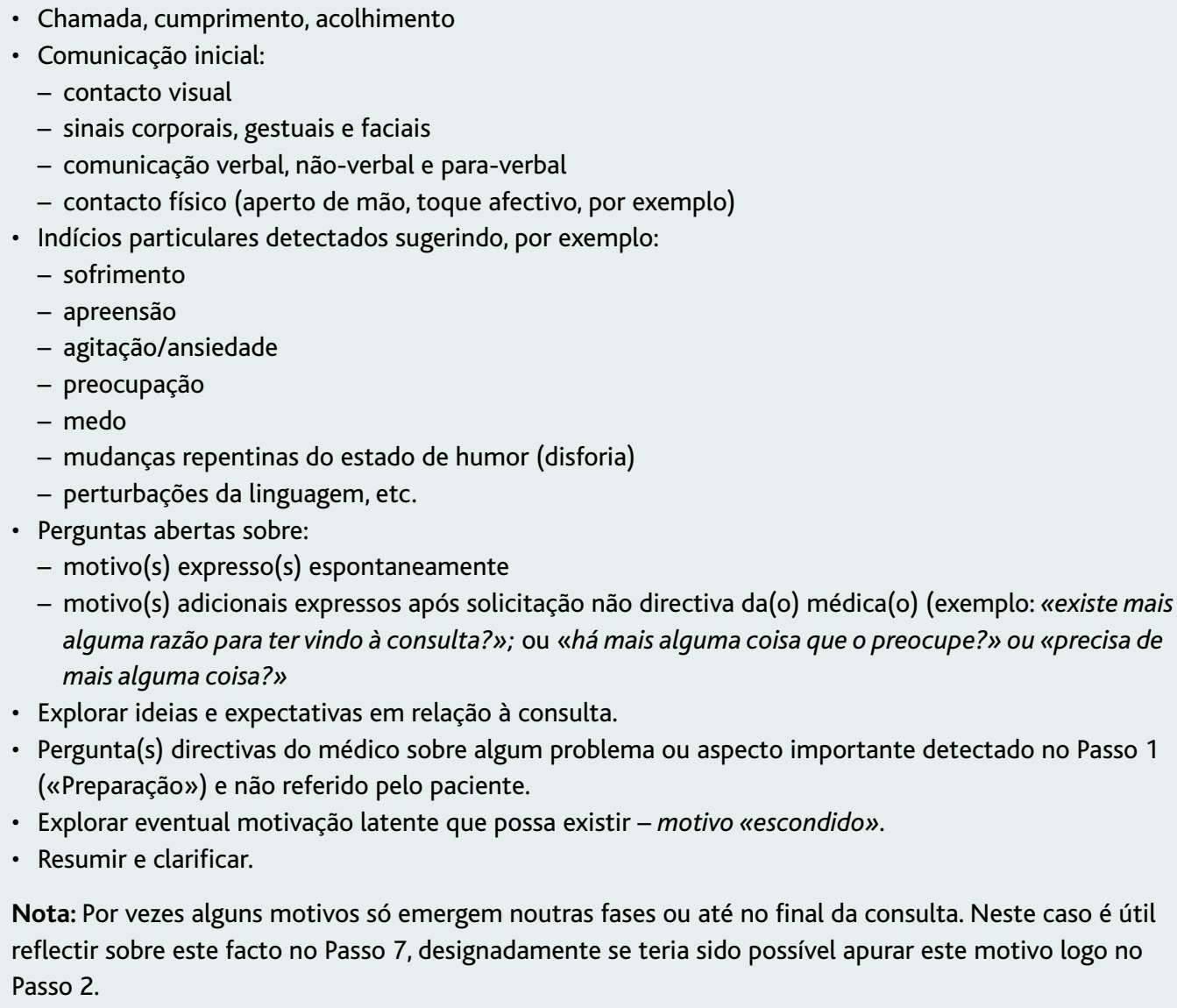 \\
\hline
\end{tabular}

\section{Passo 3 - Exploração}

Neste passo procede-se à recolha, análise e contextualização de dados e de informação subjectiva e objectiva quer biomédica quer psicossocio-cultural, através da condução criteriosa da anamnese e da realização do exame objectivo (selectivo ou geral). A informação subjectiva e objectiva assim recolhida deve ser sempre contextualizada (Quadro IV).

Os principais objectivos deste passo são:

a) obter uma anamnese adequada tendo em conta os motivos, as expectativas e a «agenda» acordada para esta consulta;

b) realizar o exame objectivo adequado à caracterização dos problemas em estudo; c) testar hipóteses;

d) complementar e contextualizar a informação recolhida, tendo em conta as dimensões biomédica e psicossocio-cultural do paciente e dos problemas abordados; e) integrar e resumir os dados e a informação recolhidos.

\section{Passo 4 - Avaliação}

Este passo é dedicado à interpretação dos dados e da informação recolhidos, à formulação de diagnósticos, à elaboração de explicação ou explicações que façam sentido para o paciente, bem como à abordagem de aspectos de previsão ou prognóstico e à avaliação dos impactos na qualidade de vida (Quadro V). É altura de inter-relacionar os dados e a informação recolhidos e de 


\section{QUADRO IV. Passo 3 - Exploração}

\begin{tabular}{|c|c|}
\hline Exercício & $\begin{array}{l}\text { - Condução criteriosa da anamnese e realização do exame objectivo (selectivo ou geral) para recolha, } \\
\text { análise e contextualização de dados e de informação biomédica e psicosocio-cultural em relação aos } \\
\text { problemas abordados. }\end{array}$ \\
\hline $\begin{array}{l}\text { Aspectos a ter } \\
\text { em conta }\end{array}$ & $\begin{array}{l}\text { - Explorar sintomas (dimensão biomédica e eventual modulação psicosociocultural): } \\
\text { - identificar sintomas/sinais/outra informação, em especial os que têm alto valor preditivo de } \\
\text { gravidade de uma situação, ou quanto ao grau de certeza do diagnóstico } \\
\text { - atender a «significados», a «interpretações», a «explicações», às «crenças» e às «representações» } \\
\text { das queixas e sintomas, para o paciente } \\
\text { - avaliar os sentimentos e as emoções do paciente relativamente ao(s) seu(s) problema(s) e situação } \\
\text { de saúde } \\
\text { - avaliar o impacto que a situação de saúde tem na vida pessoal, familiar e sócio-ocupacional do } \\
\text { paciente (qualidade de vida) } \\
\text { - Exame objectivo (selectivo ou geral) } \\
\text { - Identificar factores de risco para a saúde } \\
\text { - Testar hipóteses e contextualizar os dados e a informação recolhidos } \\
\text { - Integração, síntese e resumo da informação recolhida }\end{array}$ \\
\hline
\end{tabular}

\section{QUADRO V. Passo 4 - Avaliação}

Exercício

\section{Aspectos a ter} em conta
- Inter-relacionar os dados e a informação recolhidos e integrá-los num quadro interpretativo e preditivo coerente que concilie duas dimensões:

a) racionalidade técnico-científica médica

b) explicação ou explicações que façam sentido para o paciente e tenham em conta as suas preocupações e a sua cultura (ideias, crenças, etc.).

- formulação de diagnósticos

- explicação e sentido para o paciente

- previsão/prognóstico

- avaliação de impactos na funcionalidade e na qualidade de vida do paciente

- Síntese avaliativa da situação geral de saúde da pessoa, enquadrada no seu contexto familiar (eventualmente usando instrumentos de avaliação familiar) e sócio-ocupacional

- Lista/mapa/diagrama dos problemas abordados, organizados segundo as respectivas afinidades, interrelações (nexos e teias), multimorbilidade e co-morbilidades - mapa de problemas

- Explorar crenças («beliefs») e explicações do doente para o seu problema ou situação («o que é que pensa que isso seja?» «a que atribui/porque é que acha que surgiu esse problema»?)

- «Devolver» ao paciente uma explicação da sua situação de saúde que seja suficientemente clara, tecnicamente correcta e que faça sentido para ele(a)

- Procurar o encontro das duas «agendas» e mostrar ao doente como tentamos compreender o que ele sente («empatia»)

- Fornecer a informação prognóstica possível, que for relevante para o paciente

- Avaliar os impactos sobre a funcionalidade e a qualidade de vida

- Realizar um balanço sucinto do tipo SWOT («strenghts»; «weaknesses»; opportunities»; «threats»), centrado no paciente e com o envolvimento deste - como preparação para o Plano e para a acção 
QUADRO VI. Passo 5 - Plano de cuidados e prevenção

\begin{tabular}{|c|c|}
\hline Exercício & $\begin{array}{l}\text { - Formular propostas e chegar a acordo sobre o plano de cuidados } \\
\text { - Promover a participação, o envolvimento e a responsabilização do paciente nas acções, objectivos } \\
\text { e prazos estabelecidos e data de nova consulta }\end{array}$ \\
\hline $\begin{array}{l}\text { Aspectos a ter } \\
\text { em conta }\end{array}$ & $\begin{array}{l}\text { - Propor, eventualmente, exames auxiliares de diagnóstico e explicar a respectiva natureza e justificação } \\
\text { - Justificar sucintamente o plano terapêutico proposto, a importância da auto-vigilância, etc., com base } \\
\text { em dados de prognóstico (como é que uma dada intervenção influencia ou modifica o curso da história } \\
\text { natural da doença ou situação em causa?). } \\
\text { - Propor medidas não farmacológicas, sempre que for adequado (modificações comportamentais, } \\
\text { alimentares, etc.) } \\
\text { - Explicar a terapêutica farmacológica proposta e as medidas de precaução quanto a possíveis efeitos } \\
\text { secundários } \\
\text { - Incentivar a participação do paciente no plano de cuidados - procurar compromisso do paciente em } \\
\text { - Negsumir responsabilidade e controlo sobre o seu processo de cuidados } \\
\text { - Promover, sempre que possível e adequado, medidas para tornar o doente competente para se } \\
\text { auto-cuidar (capacitação e empoderamento do paciente) } \\
\text { - Recapitular os objectivos terapêuticos a atingir e o plano sucinto de follow-up - envolvimento da } \\
\text { equipa de saúde } \\
\text { - Verificar a oportunidade de incluir alguma prevenção «oportunista», isto é, não relacionada com o } \\
\text { conteúdo essencial da consulta e com as preocupacões actuais do paciente (o «iá agora» do médico) }\end{array}$ \\
\hline
\end{tabular}

os integrar num quadro interpretativo e preditivo coerente que concilie duas dimensões:

a) a racionalidade técnico-científica da abordagem médica;

b) a produção de explicação ou explicações que façam sentido para o paciente e tenham em conta a sua cultura (ideias, crenças, etc.), as suas preocupações, o seu modo de ver e de viver a sua situação.

Para além das listas, dos diagramas, dos mapas de problemas, das avaliações fisiopatológicas e das explicações dos diferentes problemas e da situação clínica de cada pessoa, é de especial relevância avaliar as respectivas repercussões ou impactos na funcionalidade e na qualidade de vida de cada pessoa. Há várias escalas de avaliação de estados funcionais e de qualidade de vida relacionada com a saúde que podem, eventualmente, ser úteis e usadas com este propósito. ${ }^{34-37}$

\section{Passo 5 - Plano de cuidados}

O Passo 5 corresponde à definição do plano de cuidados, que inclui a realização de eventuais exames auxiliares de diagnóstico, a proposta de modificação de comportamentos alimentares ou outros, a utilização de medicamentos, o recurso a avaliações subespecializadas, etc. Sempre que possível e oportuno, podem e devem incluir-se propostas de prevenção «oportunística», o «já agora» do médico (Quadro VI).

São objectivos deste passo:

a) Formular propostas e conseguir chegar a acordo sobre o plano de cuidados e propor medidas de prevenção;

b) Promover o envolvimento e a responsabilização do paciente nas acções e nos objectivos acordados.

A medicina geral e familiar lida com todo o tipo de problemas, em todas as fases da vida dos indivíduos e das famílias. Frequentemente, lida com casos de multimorbilidade e de polimedicação, em situações muito complexas. A percentagem de situações em que é possível aplicar directamente a evidence-based medicine (EBM) é relativamente restrita. Mesmo assim, é importante recorrer sempre a este precioso apoio à decisão clínica e combiná-lo com o recurso judicioso a todas as fontes de informação e conhecimento válidos disponíveis, incluindo as do próprio paciente. ${ }^{38-43}$ 


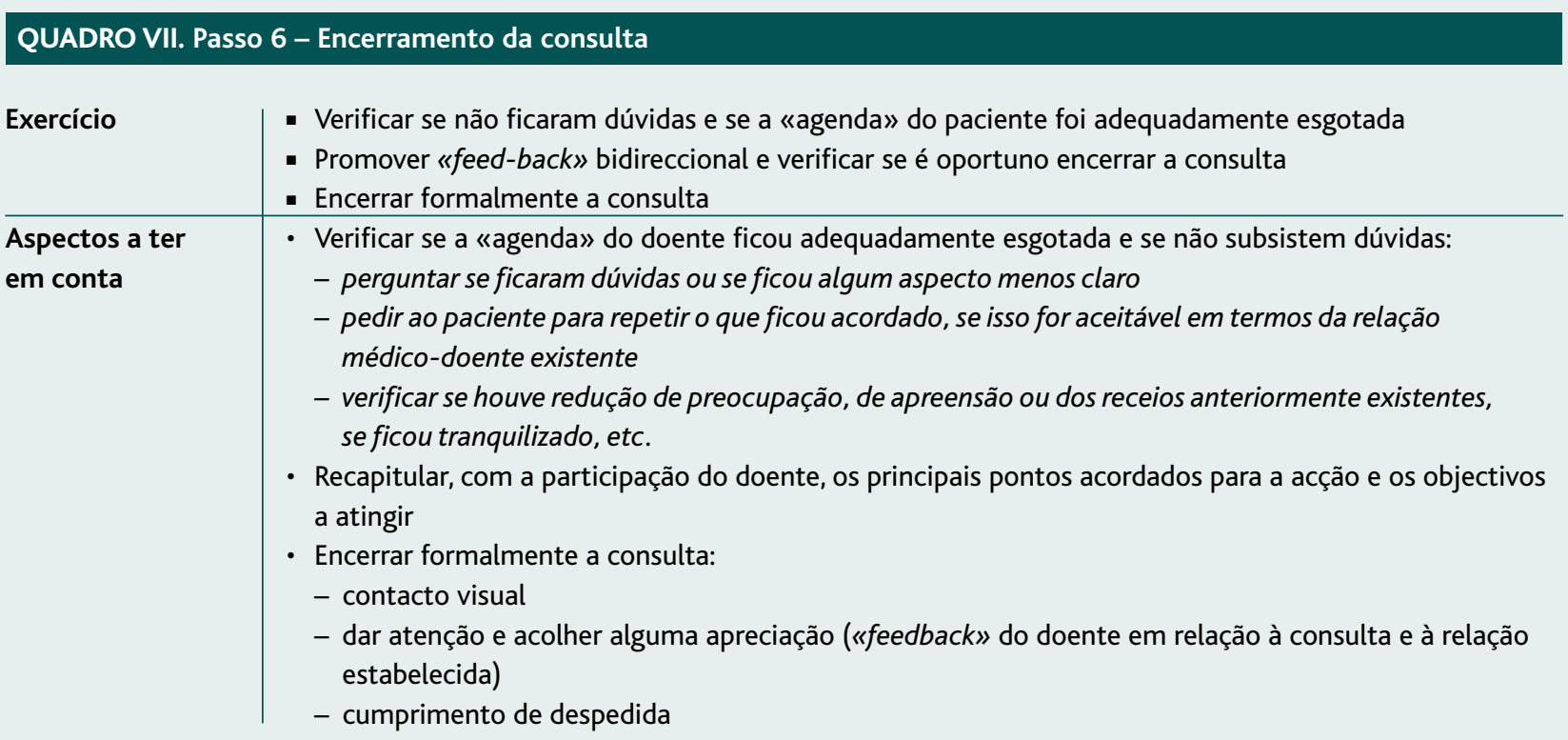

\section{QUADRO VIII. Passo 7 - Reflexão e notas finais}

\begin{tabular}{l|l} 
Exercício & - Reflectir sobre os factos ocorridos e o processo da consulta \\
\hline Aspectos a ter & - Analisar e completar os registos efectuados \\
em conta & - Ponto(s) forte(s) da consulta \\
& - Ponto(s) fraco(s) da consulta \\
- Aspecto(s) a melhorar em próximas oportunidades \\
- O que é que de mais importante ou saliente há a reter desta consulta (por exemplo: a principal \\
preocupação do paciente foi tida devidamente em conta; o que é que não se sabia e ficou a saber-se \\
nesta consulta sobre o paciente, etc.) \\
- Notas prospectivas (ideias, propostas e acções a contemplar em próximos contactos e consultas)
\end{tabular}

\section{Passo 6 - Encerramento da consulta}

Serve este passo para verificar se tudo correu como desejado e se é oportuno encerrar a consulta, se está tudo «O.K.», tanto para o médico como para o paciente (Quadro VII). Neste passo podem considerar-se como objectivos:

a) Confirmar que o plano acordado ficou claro e compreendido;

b) Identificar e resolver alguma dúvida que subsista;

c) Verificar que a «agenda» do paciente foi adequadamente esgotada;

d) Verificar se foram satisfeitas as principais expectativas;

e) Encerrar formalmente a consulta.
Passo 7 - Reflexão e notas finais

Este passo (Quadro VIII) serve para:

a) identificar e, eventualmente, rever e completar os registos efectuados nas componentes e anotar algum alerta ou aspecto a não esquecer na próxima consulta;

b) reflectir sobre os factos ocorridos e o processo da consulta, pontos fortes, pontos fracos, aspectos a melhorar;

c) identificar ganhos e avanços para o paciente e para o médico;

d) escrever eventuais notas prospectivas ou «memorando» a ter em conta nas próximas consultas;

e) identificar lacunas no conhecimento médico para colmatar. 


\section{CONCLUSÃO}

O exercício proposto neste artigo emergiu de uma necessidade sentida para estruturar e apoiar o treino e a avaliação de desempenho de internos, na execução de consultas de MGF. Tem sido testado e aperfeiçoado na prática, desde 2005, e pode ser utilizado como instrumento de apoio ao aperfeiçoamento contínuo de capacidades e de competências para a realização de consultas por parte de estudantes de medicina, de internos e de médicos de família ou de outras especialidades.

Espera-se, também, que inspire a investigação e a realização de estudos na área da consulta em MGF.

\section{REFERÊNCIAS BIBLIOGRÁFICAS}

1. Pendleton $D$, Schofield $T$, Tate P, Havelock P. The consultation: an approach to learning and teaching. Oxford: Oxford University Press; 1984.

2. Pendleton D, Schofield T, Tate P, Havelock P. The new consultation: developing doctor-patient communication. Oxford: Oxford University Press; 2003.

3. Winefield HR, Murrell TG, Clifford J. Process and outcomes in general practice consultations: problems in defining high quality care. Soc Sci Med 1995 Oct; 41 (7): 969-75.

4. Balint M. O médico o seu doente e a doença. Lisboa: Climepsi Editores, 1998. (tradução portuguesa do original: Balint M. The doctor, the patient and the illness. London: Tavistock Publications; 1957, revised 1964).

5. Salinski J. Médicos com emoções: identificar e evitar comportamentos defensivos na consulta. Amadora: Fundação Grünenthal; 2004.

6. Brandão J. Relação médico-doente: sua complexidade e papel dos grupos Balint. Rev Port Clin Geral 2007 Nov-Dez; 23 (6): 733-44.

7. Balint $E$, Norell JS. Seis minutos para o paciente: estudos sobre as interacções na consulta de clínica geral. Lisboa: Climepsi Editores; 1995.

8. Livesey PG. Partners in care: the consultation in general practice. London: William Heinemann Medical Books; 1986.

9. Borrell i Carrió F. Entrevista clínica. Manual de estratégias práticas. Barcelona: SemFyc; 2004.

10. Marvel MK, Doherty WJ, Weiner E. Medical interviewing by exemplary family physicians. J Fam Pract 1998 Nov; 47 (5): 343-8.

11. Elwyn G. Arriving at the postmodern medical consultation. Eur J Gen Pract 2004; 10 (3): 93-7.

12. MatalonA, Rabin S. Behind the consultation: reflective stories from clinical practice. Abingdon, Oxon: Radcliffe Publishing; 2007.

13. Leal I. A entrevista psicológica: técnica, teoria e clínica. Lisboa: Fim de Século Edições; 2008.

14. Neighbour R. The inner consultation: how to develop an effective and intuitive consultation style. Newbury: Petroc Press; 1999.

15. Tavares JF. A consulta consultada. Lisboa: Editora Temática; 1999.

16. Usherwood T. Understanding the consultation: evidence, theory and practice. Buckingham: Open University Press; 1999.

17. Nunes JM. Comunicação em contexto clínico. Lisboa: Bayer Health Care; 2007.

18. Byrne P, Long B. Doctors talking to patients: a study of the verbal be- haviours in the consultation. London: HMSO; 1976.

19. Ramos V. A consulta em 7 passos. Lisboa:VFBM Comunicação; 2008.

20. Engel GL. The need for a new medical model: a challenge for biomedicine. Science 1977 Apr 8; 196 (4286): 129-36.

21. McWhinney IR. Manual de Medicina Familiar. Lisboa: Inforsalus; 1994.

22. Stewart M, Brown JB, Weston WW, McWhinney IR, McWilliam CL, Freeman TR. Patient-centered medicine: transforming the clinical method. 2nd ed. Thousand Oaks, CA: SAGE Publications; 2003.

23. Stewart M, Brown JB, Donner A, McWhinney IR, Oates J, Weston WW, et al. The impact of patient-centered care on outcomes. J Fam Pract 2000 Sep; 49 (9): 796-804.

24. Tudela M, Lobo FA, Ramos V. Desafios da complexidade em medicina geral e familiar. Rev Port Clin Geral 2007 Nov-Dez; 23 (6): 715-25.

25. Morin E. Introduction à la pensée complexe. Paris: ESF; 1990.

26. Mariotti H. Complexidade e pensamento complexo: breve introdução e desafios actuais. Rev Port Clin Geral 2007 Nov-Dez; 23 (6): 727-31.

27. Mariotti H.A dança dos conceitos: In: As paixões do ego: complexidade, política e solidariedade. São Paulo: Palas Athena; 2000. p. 83-121.

28. Griffiths F, Byrne D. General practice and the new science emerging from the theories of «chaos» and complexity. Br J Gen Pract 1998 Oct; 48 (435): 1697-9.

29. Zimmerman B, Lindberg C, Plsek P. Edgeware: insights from complexity science for health care leaders. 2nd ed. Irving, TX:VHA, 2001.

30. Rowe A, Hogarth A. Use of complex adaptive systems metaphor to achieve professional and organizational change. J Adv Nurs 2005 Aug; 51(4): 396-405.

31. Beckman HM, Frankel RM. The effect of clinician behavior on the collection of data. Ann Intern Med 1984 Nov; 101 (5): 692-6.

32. Marvel KM, Epstein RM, Flowers K, Beckman HB. Soliciting the patient's agenda: have we improved? JAMA 1999 Jan 20; 281 (3): 283-7.

33. Gray JA. The resourceful patient. Oxford: eRosetta Press; 2002.

34. Wilkin D, Hallam L, Doggett MA. Measures of need and outcome for primary Health Care. Oxford: Oxford University Pres; 1992.

35. Broeiro P, Ramos V, Tavares I, Cunha E, Amorim J. Avaliação de estados funcionais no idoso: exercício de aplicação de uma versão portuguesa da escala COOP/WONCA Charts. Acta Med Port 1995 Maio; 8 (5): 279-88.

36. Ferreira PL. Criação da versão portuguesa do MOS SF-36. Parte I: adaptação cultural linguística. Acta Med Port 2000 Jan-Abr; 13 (1-2): 55-66.

37. Ferreira PL. Criação da versão portuguesa do MOS SF-36. Parte II: testes de validação. Acta Med Port 2000 Maio-Jun; 13 (3): 119-27.

38. Starfield B. Co-morbidity and its challenges for quality of primary care. Rev Port Clin Geral 2007 Mar-Abr; 23 (2): 179-80.

39. Santos I. O doente com patologia múltipla: co-morbilidade de quatro doenças crónicas [tese de doutoramento]. Lisboa: Universidade Nova de Lisboa, Faculdade de Ciências Médicas, 2006.

40. Gervas J, Santos I.A complexidade da co-morbilidade. Rev Port Clin Geral 2007 Mar-Abr; 23 (2): 181-9.

41. Broeiro P, Ramos V, Barroso R. O mapa de problemas - um instrumento para lidar com a morbilidade múltipla. Rev Port Clin Geral 2007 MarAbr; 23 (2): 209-15.

42. Innes AD, Campion PD, Griffiths FE. Complex consultations and the «edge of chaos». Br J Gen Pract 2005 Jan; 55 (510): 47-52.

43. Gray DP. Evidence-based medicine and patient-centred medicine: the need to harmonize. J Health Serv Res Policy 2005 Apr; 10 (2): 66-8. 


\title{
AGRADECIMENTOS
}

São devidos agradecimentos a todos os que, por diversas formas, e ao longo dos últimos quatro anos, têm contribuído para o desenvolvimento deste projecto.

\author{
ENDEREÇO PARA CORRESPONDÊNCIA \\ Vítor Ramos \\ USF Marginal (Centro de Saúde de Cascais) \\ Unidade de S. João do Estoril \\ Piso 2, Rua Dr. Egas Moniz \\ 2765-458 São João do Estoril \\ Tel. 214643720 - Fax. 214643719 \\ E-mail:vramos@ensp.unl.pt
}

\section{ABSTRACT}

This article presents a model to practice and to critical analysis of consultations in general practice/family medicine. It is a proposal to be adapted to each particular clinical situation. It begun to be developed in 2005 as a small exercise to support training in consultation skills for the family medicine vocational trainees / residents in the Cascais Health Centre, in Portugal. This exercise was further developed and became a working book, edited in Lisbon in September 2008. The model proposes a consultation structure with three phases and seven steps.

The starting phase includes two steps: preparation and the first minutes:

1 - Preparation - with a self-evaluation on how the doctor feels for the next consultation; who is the next patient? with a brief review of the clinical records; and a brief appraisal of the physical environment of the consultation office (comfort, cleanness, good order, etc.).

2 - The first minutes - this phase is dedicated to the initial listening and attentive observation to the called patient. It is when doctor and patient meet each other and get the first signs and impressions and when a caring and therapeutic climate begins to be prepared. In this phase doctor pays a special attention to patient's expectations and motivations (here and now).

An intermediate phase follows, with three steps: exploration; assessment; and plan (EAP) always actively involving the patient in the decision making process:

3 - Exploration and contextualization - a step for the anamnesis, physical examination and contextualization of the approached health problems, understanding the patient, his illness, their worries and their problems, without forcing or deforming the patient narrative.

4 - Assessment (interpretation; diagnosis; prognosis) - the step when data and information are collected, interpreted, inter-related and integrated in a coherent explanatory framework, which make sense both for the doctor and for the patient, including quality of life impact assessment.

5 - Plan for action/care - to propose, negotiate and agree on actions and aims, including, if appropriate, preventive activities.

A final phase to close the consultation and to reflect on it:

6 - Closing the consultation - with the confirmation that there are no remaining doubts and that the main expectations were met. It is also the time to accept feed-back from the patient about the consultation, namely his/her immediate satisfaction before closing the consultation warmly and formally.

7 - Final reflection - a reflexive moment on what happened during the consultation. It is also a step for «house keeping» on cognitive and emotional aspects before passing to the next patient. This phase may be useful to complete or to reformulate clinical records of the patient.

Keywords: Consultation; Family Medicine; General Medicine; Critical Appraisal of Clinical Consultation; Vocational Training. 


\section{ANEXO I}

\section{GRELHA DE OBSERVAÇÃO QUALITATIVA SUMÁRIA}

\begin{tabular}{|c|c|c|c|c|}
\hline Passo 1 - Preparação - Itens & 0 & 1 & NA & Observações \\
\hline 1. Atenção à situação do médico e do gabinete & & & & \\
\hline 2. Aspectos de personalização - próximo utente & & & & \\
\hline 3. Contexto familiar & & & & \\
\hline 4. Contexto sócio-ocupacional & & & & \\
\hline 5. Ver resumo e lista de problemas & & & & \\
\hline 6. Ver medicamentos que toma habitualmente & & & & \\
\hline 7. Ver conteúdo da última consulta & & & & \\
\hline 8. Rever outras consultas e contactos & & & & \\
\hline $\begin{array}{l}\text { 9. Identificação de omissões, aspectos a clarificar, } \\
\text { a explorar, anotar, «lembretes», etc. }\end{array}$ & & & & \\
\hline Passo 2 - Primeiros minutos - Itens & 0 & 1 & NA & Observações \\
\hline 10. Observação atenta e cumprimento inicial & & & & \\
\hline 11. Atenção a indícios particulares & & & & \\
\hline 12. Motivo(s) expressos no início & & & & \\
\hline 13. Motivo(s) adicionais & & & & \\
\hline 14. Motivos latentes? («ocultos») & & & & \\
\hline 15. Exploração de ideias, expectativas e objectivos do paciente & & & & \\
\hline $\begin{array}{l}\text { 16. Foi lembrado algum problema identificado no Passo } 1 \\
\text { e não referido pelo paciente? }\end{array}$ & & & & \\
\hline Passo 3 - Exploração - Itens & 0 & 1 & NA & Observações \\
\hline $\begin{array}{l}\text { 17. Sintomas, em especial sintomas com alto valor } \\
\text { preditivo (diagnóstico e gravidade) }\end{array}$ & & & & \\
\hline 18. Emoções, sentimentos, pontos de vista & & & & \\
\hline 19. Repercussões na qualidade de vida & & & & \\
\hline 20. Factores de risco & & & & \\
\hline 21. Preparação/consentimento para o ex. objectivo & & & & \\
\hline 22. Exame objectivo (geral ou mais focalizado) & & & & \\
\hline 23. Síntese - resumo & & & & \\
\hline Passo 4-Avaliação - Itens & 0 & 1 & NA & Observações \\
\hline 24. Avaliação sucinta - informação ao paciente & & & & \\
\hline 25. Lista / mapa / diagramas dos problemas & & & & \\
\hline 26. Exploração de crenças, de explicações e de dúvidas do paciente & & & & \\
\hline 27. Explicação apresentada pelo médico & & & & \\
\hline 28. Encontro de agendas (médico e paciente) & & & & \\
\hline 29. Aspectos de previsão/prognóstico & & & & \\
\hline 30. Aval. funcionalidade e qualidade de vida & & & & \\
\hline 31. Balanço sucinto «SWOT» - «pré-acção» & & & & \\
\hline
\end{tabular}




\begin{tabular}{|c|c|c|c|c|}
\hline Passo 5 - Plano - Itens & 0 & 1 & NA & Observações \\
\hline \multicolumn{5}{|l|}{ 32. Justificação do pedido de EAD } \\
\hline \multicolumn{5}{|l|}{ 33. Explicação de medidas não farmacológicas } \\
\hline \multicolumn{5}{|l|}{ 34. Explicação de prescrições farmacológicas } \\
\hline \multicolumn{5}{|l|}{ 35. Negociação, participação e envolvimento do paciente } \\
\hline \multicolumn{5}{|l|}{ 36. Definição participada de objectivos e compromissos } \\
\hline \multicolumn{5}{|l|}{ 37. Aspectos de capacitação e empoderamento } \\
\hline \multicolumn{5}{|l|}{ 38. «Follow-up» acordado } \\
\hline \multicolumn{5}{|l|}{ 39. Prevenção «oportunística» abordada e outra } \\
\hline Passo 6 - Encerramento - Itens & 0 & 1 & NA & Observações \\
\hline \multicolumn{5}{|l|}{$\begin{array}{l}\text { 40. Confirmação de que houve clareza e compreensão adequada } \\
\text { quanto ao acordado }\end{array}$} \\
\hline \multicolumn{5}{|l|}{ 41. Foram exploradas eventuais dúvidas? } \\
\hline \multicolumn{5}{|l|}{ 42. A «agenda» do doente foi esgotada? } \\
\hline \multicolumn{5}{|l|}{$\begin{array}{l}\text { 43. Foram recapituladas as principais acções na sequência } \\
\text { da consulta? }\end{array}$} \\
\hline \multicolumn{5}{|l|}{ 44. Encerramento formal da consulta } \\
\hline Passo 7 - Reflexão final - Itens & 0 & 1 & NA & Observações \\
\hline \multicolumn{5}{|l|}{ 45. Registos efectuados - completar registos } \\
\hline \multicolumn{5}{|l|}{ 46. Pontos fortes desta consulta } \\
\hline \multicolumn{5}{|l|}{$\begin{array}{l}\text { 47. Pontos fracos desta consulta em relação ao desempenho } \\
\text { do médico }\end{array}$} \\
\hline \multicolumn{5}{|l|}{ 48. Aspecto(s) a melhorar (desempenho médico) } \\
\hline \multicolumn{5}{|l|}{ 49. O que houve de importante a reter? } \\
\hline \multicolumn{5}{|l|}{$\begin{array}{l}\text { 50. Notas prospectivas - lembrar e anotar algum «alerta» ou } \\
\text { «recordatório» a ter em conta na próxima consulta }\end{array}$} \\
\hline \multicolumn{5}{|l|}{ 51. O que aprendi/ganhei em termos pessoais e profissionais? } \\
\hline $\begin{array}{l}\text { 52. O que devo estudar / consultar / ou rever a propósito de } \\
\text { algum problema do paciente? }\end{array}$ & & & & \\
\hline
\end{tabular}

